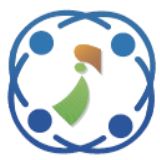

\title{
A Modified Compressed Sensing-Based Decomposition Approach to Analyze Electrodermal Activity Signals Using Matrix-Free Convex Optimization
}

\author{
Sudarsan Prabhakaran ${ }^{1 *}$ \\ Niranjil Kumar Ayyamperumal ${ }^{2}$ \\ ${ }^{I}$ Department of Electronics \& Communication Engineering, \\ Aishwarya College of Engineering \& Technology, Erode, Tamilnadu, India \\ ${ }^{2}$ Department of Electronics and Communication Engineering, \\ Dhanalakshmi College of Engineering, Tambaram, Chennai, Tamilnadu, India \\ * Corresponding author's Email: sudarsanphd123@ gmail.com
}

\begin{abstract}
In modern decades, a human socioeconomic lifestyle has been disrupted by their stress levels. Normally, the stress levels were measured based on the Electrodermal Activity (EDA) analysis that analyses the changes in the Skin Conductance Response (SCR) level. As a result, different sensors were designed to analyze the changes in human emotional levels using the EDA signals. During analysis, the unwanted noise components in the signals were removed by using a Compressed Sensing-based Decomposition (CSD) approach. Conversely, this approach has high computational complexity. Therefore in this article, a Modified CSD (MCSD) for EDA signal is proposed that may vary the shape of the impulse response with time and varied noise models. The key aim of this model is to improve the recovery accuracy of EDA signal decomposition and enhance the human stress monitoring system. In this modified model, a computationally efficient decomposition method is proposed by using matrix-free convex optimization modeling that exploits the Toeplitz structure to allow decomposition of EDA signals with provable bounds on the recovery of true SCR events by using the computationally efficient algorithm and guarantee a better recovery accuracy. Finally, this approach is tested on Wearable Stress and Affect Detection (WESAD) dataset to exhibit that the proposed MCSD approach achieves a recovery accuracy of $92.4 \%$ which is $2.55 \%$ higher than the existing CSD approach.
\end{abstract}

Keywords: Human stress monitoring, Electrodermal activity, Compressed sensing-based decomposition, Matrixfree convex optimization, Toeplitz structure.

\section{Introduction}

Emotion or human stress monitoring can improve the quality of life in different situations. Nowadays, multi-modal emotion recognition systems and devices such as smart watches have been built that measure physiological signals and significantly smaller and more affordable. Many researchers have tried for acquiring different data including physiological signals in order to recognize the emotions by means of a few special characteristics of the input. Generally, emotions like stress, anger, fear, etc., are controlled by the sympathetic nervous system. Electrodermal Activity (EDA) refers to a phenomenon where the changes in emotion levels of a person directly reflect their psychological state [1]. EDA is also known as Skin Conductance Level (SCL), SC Response (SCR), ED Response (EDR), etc., and can provide a noninvasive, easily acquired, robust and economical approach for recording physiological data. If the sympathetic part of a regular nervous system is greatly produced, then sweat gland activities will also be increased with increasing the SC. According to this condition, an SC refers a measure of an emotional and sympathetic response. Most of the researchers recommended that the EDA has more difficult than it seems and research extends into the source and effect of EDA. Skin resistance was one of the key measurements for medical diagnosis that involve a pair of electrodes in contact within the 
skin and passing a small amount of current causing a voltage drop which differs with different skin resistance while subjected to various stimuli. Usually, it is influenced by several cognitive, emotional and motor tasks. Mostly, the EDA analysis approaches have been focused on two major features of the physiological signal, namely phasic and tonic [2].

Principally, fast varying activity is associated with the phasic feature of the signal whereas the tonic feature associates with slow changes over time. Over the past decades, different EDA analysis-based human stress monitoring system has been designed by analyzing the physiological parameters such as heart rate, skin conductivity, respiration rate, electrocardiogram, skin temperature and electroencephalography data. Among different methods, Jain et al. [3] proposed a Compressed Sensing-based Decomposition (CSD) of EDA signals to alleviate the effects of unwanted noise components and exposing the underlying physiological signal. By using the compressed sensing-based decomposition with pre-processing, the baseline signal was modeled and facilitated to recover the user's responses. However, this approach has high computational complexity.

Hence in this article, a Modified CSD (MCSD) model for EDA signal is proposed that may vary the shape of the impulse response with time and varied noise models. The main objective of this model is to improve the recovery accuracy of EDA signal decomposition and enhance the human stress monitoring system. In this model, a computationally efficient decomposition method is developed by using matrix-free convex optimization modeling that exploits the Toeplitz structure to guarantee better recovery accuracy. Thus, it allows an accurate decomposition of EDA signals with provable bounds on the recovery of user responses.

The rest of the article is structured as follows: Section 2 presents the literature survey related to the human stress monitoring based on EDA analysis. Section 3 explains the proposed methodology. Section 4 illustrates the experimental results of the proposed method. Finally, Section 5 concludes the research work.

\section{Related work}

Finset et al. [4] analyzed EDA to empathic statements in clinical interviews with fibromyalgia patients. In this analysis, patients with fibromyalgia were invited to a clinical interview in a randomized manner in which interviewer empathic or inattentive character and the emotional or neutral content of the interview were manipulated. Moreover, EDA was measured during the interview. Additionally, the patient's after state and patient satisfaction were also assessed. However, clinicians must be aware of finding empathic statements.

Kappeler-Setz et al. [5] proposed a promising sensor system with the aim of for long-term monitoring of both physiological and behavioural information associated with the bipolar disorder patients as well providing a better diagnosis of bipolar disorder. A correlation between EDA measurements at the fingers and feet was analyzed. In addition, the influence of emotional stimulus was evaluated. However, the effect of specific emotions of human using EDA signals was not analyzed.

Sano et al. [6] presented a quantitative analysis of wrist EDA patterns during sleep by using dry electrodes. In this analysis, EDA paterns were automatically measured in healthy adults based on forearm SCR and actigraphy during sleep. The thresholds were systematically compared for automatically detecting EDA peaks and also the criteria for EDA storms were established. However, further improvement was needed to explain the influences of EDA patterns.

Chaspari et al. [7] proposed a sparse representation of EDA with the help of knowledgedriven dictionaries. Initially, EDA-specific dictionaries were built to exactly model both slowvarying tonic part and the signal fluctuations known SCR. Then, the greedy sparse representations were used to decompose the signal into a small number of tonic and phasic atoms from the dictionary. At last, the SCR occurring in the signal was identified automatically by post-processing the chosen phasic atoms. However, this technique has high computational complexity.

Xia et al. [8] proposed active learning for EDA classification that reduces the labeling effort required to train a machine learning algorithm in signal classification. However, feature extraction and selection were computationally expensive. Greco et al. [9] proposed a convex optimization approach, maximum posterior probability and sparsity for analyzing the EDA. In this model, SC was described as the sum of three terms such as the phasic component, the tonic component and an additive white Gaussian noise including EDA measurement errors and artifacts. However, the computational complexity was high.

Ghaderyan \& Abbasi [10] proposed an efficient automatic workload estimation method based on the EDA using pattern classifier combinations. In this method, three different decomposition techniques such as Fourier, cepstrum and wavelet transforms 
were proposed for analyzing the EDA. Also, the efficiency of different statistical and entropic features was discussed and compared. The features were processed by Principal Component Analysis (PCA) and machine-learning techniques for recognizing different levels of an arithmetic process. Moreover, these methods were added into a workload estimation system according to the feature-level and decision-level combinations. However, further improvement was required to discriminate the levels of emotional arousal.

Giromini et al. [11] presented a study on EDA during stress to investigate the predictive validity of the Rorschach Performance Assessment System (RPAS) variables from the stress and distress domain. This investigation was done by testing whether they predicted increased sympathetic reactivity to a mild, laboratory-induced stress, occurred one week after Rorschach administration. In this study, a small student sample was contributed and the first meeting (T1) participants were administrated to the Rorschach process according to the R-PAS guidelines. After a week (T2), their EDA was recorded during exposure to a mild laboratory stress-inducing process. According to this, the stress and distress R-PAS variables measured at T1 was positively correlated with an increased sympathetic reactivity to stress at $\mathrm{T} 2$ that indicates the greater EDA changes from baseline to stress and recovery. However, few Rorschach scores were not considered. Melander et al. [12] explored the identification of agitation in individuals with dementia by measuring EDA. In this system, a binary logistic regression model was applied for measuring the relationship between the sensor and the assistant nurse's structured observations of agitation. However, more detailed observations were neglected. Hwang et al. [13] proposed a method based on the EDA fluctuations during sleep-wake transitions. In this model, each person's EDA signals were measured at the middle and ring fingers of the dominant hand during polysomnography for estimating the sleep period time. However, the detection of short periods of wakefulness or arousal was not accurate.

Zangróniz et al. [14] designed a novel and unobtrusive wearable monitoring device based on the EDA in order to identify an individual distress condition from the captured physiological signals. Initially, a lightweight wearable device was placed on the wrist of an individual that facilitate the continuous physiological measurements. Then, the signals were gathered and processed for extracting the most significant features. Finally, the calm or distress condition was identified by classifying the extracted features by using statistical analysis. However, the predictions were not being generalized directly to the total population.

\subsection{Overview of existing models}

\section{i) Sparse Decomposition Model}

In the sparse decomposition model [7], sparse representation of a signal $f \in \mathfrak{K}^{L}$ was defined by the nonconvex problem as:

$$
\min _{c}\|c\|_{0} \text { Subject to } f=D c
$$

In Eq. (1), $D \in \mathfrak{K}^{L \times Q}$ denotes an over-complete dictionary matrix with $Q$ prototype signal-atoms and $c \in \mathfrak{K}^{Q}$ denotes the atom coefficients with $N \ll Q$ nonzero elements. After the first iteration, signal residual $R f$ is decomposed by chosen an atom from $D$ that best matches its structure. The residual $R^{n+1} f$ was defined as:

$$
R^{n+1} f=R^{n} f-\left(\left(R^{n} f\right)^{T} g_{\zeta_{n}}\right) \cdot g_{\zeta_{n}}
$$

Here, $\quad g_{\zeta_{n}} \in D \quad$ was chosen to maximize $\left(R^{n} f\right)^{T} g_{\zeta_{n}}$. After $N$ iterations, the actual signal $f$ was approximated by $f_{N}$ :

$$
\begin{gathered}
f_{N}=\sum_{n=0}^{N-1}\left(\left(R^{n} f\right)^{T} g_{\zeta_{n}}\right) \cdot g_{\zeta_{n}} \\
=\sum_{n=0}^{N}\left[\delta_{n} \cdot\left(\left(R^{n} f\right)^{T} g_{\beta_{n}}\right) \cdot g_{\beta_{n}}+\left(1-\delta_{n}\right) \cdot\right. \\
\left.\left(\left(R^{n} f\right)^{T} g_{\gamma_{n}}\right) \cdot g_{\gamma_{n}}\right]
\end{gathered}
$$

In Eq. (3), $\delta_{n} \in\{0,1\}$ chooses either a tonic or a phasic atom, $\beta_{n} \in \mathbb{B}$ and $\gamma_{n} \in \Gamma^{(1)}$ or $\gamma_{n} \in \Gamma^{(2)}$ or $\gamma_{n} \in \Gamma^{(3)}$ were the parameters of $n^{\text {th }}$ atom. Then, the phasic atoms were grouped according to their location with a histogram of $p_{n}$ values to capture one SCR as:

$$
S C R=\sum_{\left\{g_{\gamma_{n}}\right\}} c_{n} g_{\gamma_{n}}
$$

Thus, the SCR detection was achieved; but, the computational complexity was high.

\section{ii) Convex Optimization Model}

In the cvxEDA model [9], consider the $N$-sample SC signal $(y)$ as the sum of a tonic $(t)$ and a phasic $(r)$ component plus an additive noise $(\epsilon)$ :

$$
y=M q+B l+C d+\epsilon
$$


In Eq. (5), $q$ denotes an auxiliary variable, $l$ denotes the vector of spline coefficients, $d$ represents a $2 \times 1$ vector with the offset and slope coefficients for the linear drift, $B$ refers to the tall matrix whose columns are cubic $B$-spline basic functions, $C$ refers to a $N \times 2$ matrix with $C_{i, 1}=1$ and $C_{i, 2}=i / N$ and $M$ is a tridiagonal matrix with elements $M_{i, 1}=M_{i, i-2}=1, M_{i, i-1}=2,3 \leq i \leq N$. Given this observation model, the aim was identifying the Maximum A Posteriori (MAP) spike train $p$ and $t$ parameterized by $[q, l, d]$ for $y$ :

$$
[q, l, d]=\underset{q, l, d}{\operatorname{argmax}} P[q, l, d \mid y]
$$

The optimization problem of this model was defined as:

$$
\begin{gathered}
\min \frac{1}{2}\|M q+B l+C d-y\|_{2}^{2}+\alpha\|A q\|_{1}+\frac{\gamma}{2}\|l\|_{2}^{2} \\
\text { Subjected to } A q \geq 0
\end{gathered}
$$

This optimization problem was solved by using the sparse-QP solvers. Once the optimal $[q, l, d]$ was obtained, the tonic component $t$ and phasic component $p$ can be derived as:

$$
t=B l+C d ; p=A q
$$

In this optimization problem, the objective function to be minimized was a quadratic measure of misfit between the predicted and the observed data. However, the computational complexity of this model was high.

\section{iii) Compressed Sensing-based Decomposition (CSD) Model}

The SCR events signal detection was achieved by decomposing the EDA observation signal based on the compressed sensing method [3]. The observed signal $s_{o}$ was linearly transformed by using the difference matrix $A$ which has Toeplitz matrix $T_{h}$ constructed from a vector $h \in \mathbb{R}^{T}$. Such that, the estimation of true SCR events signal $e$ and modified baseline signal $A s_{b}$ by solving the optimization problem using compressed sensing method. This optimization problem is known to be a convex problem which can be solved by using wellknown convex optimization software. The objective of this model was distinguished with the proposed model in Section 3.

However, the computational complexity of this method was high due to consideration of the matrix convex optimization modeling. Hence, matrix-free convex optimization modeling is proposed in this article to reduce the computational complexity and achieve the computationally efficient algorithm.

\section{Proposed methodology}

In this section, the proposed MCSD model is explained in brief. Typically, the observed EDA SC signal is characterized by two dominant components, namely tonic and phasic. The SCRs are driven by occurrences of SCR events signal. The sweating of a person may cause an SC in the form of an SCR observation. In this model, a baseline model [3] inspired by the wearable devices where changes in the locations of sensors due to mobility may lead to rapid changes in the EDA signal known as baseline shifts. Such baseline shifts are incorporated along with the SCL component into a baseline signal $s_{b}$. Specifically, the presence of the baseline $s_{b}$ may affect the process of recovering the true SCR events $e$ from the observed EDA signal $s_{o}$ is still a challenging one. This problem is often known as a de-mixing problem.

Therefore, this problem is solved by modeling the EDA signals that mitigate the effects of the baseline $s_{b}$ and noise $n$. Consider that the baseline $s_{b}$ have nearly similar consecutive components for most of the signal elements. Thus, they can be transformed to approximately sparse signals by multiplying with the pairwise difference matrix $A$ defined as:

$$
A=\left[\begin{array}{ccccc}
1 & -1 & 0 & \ldots & 0 \\
0 & 1 & -1 & \ldots & 0 \\
\vdots & \vdots & \ddots & \ddots & \vdots \\
0 & 0 & \ldots & 1 & -1
\end{array}\right]
$$

Certainly, only the observed signal $s_{o}$ is accessed. As a result, the signal $s_{O}$ is linearly transformed by using the difference matrix $A$ as follows:

$$
A s_{o}=A T_{h} e+A s_{b}+A n
$$

In Eq. (10), $T_{h}=(t+T-1) \times T$ represents a Toeplitz matrix built from an impulse response vector $h \in \mathbb{R}^{T}$ i.e., sweat response of a user and is defined as: 


$$
T_{h}=\underbrace{\left[\begin{array}{cccc}
h_{1} & 0 & \ldots & 0 \\
h_{2} & h_{1} & \vdots & \vdots \\
\vdots & \vdots & \ddots & 0 \\
h_{t} & h_{t-1} & \vdots & h_{1} \\
0 & h_{t} & \vdots & \vdots \\
\vdots & \vdots & \ddots & \vdots \\
0 & \ldots & \ldots & h_{t}
\end{array}\right]}_{\text {T columns }}
$$

Such that the convolution between vectors $h \in$ $\mathbb{R}^{T}$ and $e \in \mathbb{R}^{T}$ denoted by $h * e$ is a vector in $\mathbb{R}^{t+T-1}$ and it can be rewritten in terms of matrixvector multiplications as $h * e=T_{h} e$. Based on this transformation, the modified baseline $A s_{b}$ is approximately sparse since the structure of $s_{b} \in \mathcal{B}_{\gamma}^{c}$. Due to this sparsity, the transformed $s_{b}$ has a similar structure to the SCR events $e$. This statement is leveraged for mutually estimating $e$ and $A s_{b}$. So, the observation model can be rewritten by rearranging the terms as:

$$
A s_{o}=\left[\begin{array}{ll}
A T_{h} & I
\end{array}\right]\left[\begin{array}{c}
e \\
A s_{b}
\end{array}\right]+A n
$$

In Eq. (12), $I$ is the identity matrix, $A s_{o}$ is the modified observed signal, $A T_{h}$ is the modified Toeplitz matrix and $A n$ is the modified noise signal. This problem is transformed into estimating a vector that is approximately sparse with $l+c$ significant components in $\mathbb{R}^{t+T-2}$ where $l$ denotes the number of significant non-zero elements in $e$ and $c$ represents the number of significant non-zeros in $A s_{b}$. To estimate $e$ and $A s_{b}$, the following problem can be solved:

$$
\begin{aligned}
& \min _{\check{e} \in \mathbb{R}^{T}, \breve{u} \in \mathbb{R}^{t+T-2}}\|\tilde{e}\|_{1}+\|\tilde{u}\|_{1} \\
& \text { Subject to }\left\|A s_{o}-\left[\begin{array}{ll}
A T_{h} & I
\end{array}\right]\left[\begin{array}{l}
\tilde{e} \\
\tilde{u}
\end{array}\right]\right\|_{2} \leq \eta \\
& \text { Where } \eta>0
\end{aligned}
$$

In Eq. (13), $\tilde{e}$ is the recovered SCR events signal, $\tilde{u}$ is the recovered modified baseline $A s_{b}, \quad \eta>0$ denotes a parameter that can be selected based on the energy of noise $n$. This problem is called as a convex problem that can be solved by using convex optimization. Nonetheless, this approach has high computational complexity.

Therefore, in this proposed model, matrix-free convex optimization is applied that uses an expression Directed Acyclic Graph (DAG) data structure to represent convex optimization problems as input to the algorithm. As a result, the problem (13) can be rewritten as:

$$
\begin{gathered}
\min _{\check{e} \in \mathbb{R}^{T}, \breve{u} \in \mathbb{R}^{t+T-2}}\|\tilde{e}\|_{1}+\|\tilde{u}\|_{1} \\
\text { Subject to }\left\|A s_{o}-\left[\begin{array}{ll}
A T_{h} & I
\end{array}\right]\left[\begin{array}{l}
\tilde{e} \\
\tilde{u}
\end{array}\right]\right\|_{2} \leq \eta, \mathcal{K}
\end{gathered}
$$

In Eq. (14), $\mathcal{K}$ refers to the matrix-free convex cone. In this way, an optimization problem (14) can be assumed as:

$$
\min _{\tilde{e} \in \mathbb{R}^{T}, \tilde{u} \in \mathbb{R}^{t+T-2}} f_{0}\left(\|\tilde{e}\|_{1}+\|\tilde{u}\|_{1}\right)
$$

Subject to $f_{h}\left(\left\|A s_{o}-\left[\begin{array}{ll}A T_{h} & I\end{array}\right]\left[\begin{array}{c}\tilde{e} \\ \tilde{u}\end{array}\right]\right\|_{2}\right) \leq \eta$, $h=1, \ldots, n$ and

$$
h_{i} * \tilde{e}=T_{h} \tilde{e}, i=1, \ldots, n
$$

Here, $\quad \tilde{e} \in \mathbb{R}^{T}$ and $\tilde{u} \in \mathbb{R}^{t+T-2}$ are the optimization variables, $\quad f_{0}: \mathbb{R}^{T}\left(\mathbb{R}^{t+T-2}\right) \rightarrow$ $\mathbb{R}, \ldots, f_{h}: \mathbb{R}^{T}\left(\mathbb{R}^{t+T-2}\right) \rightarrow \mathbb{R}$ are convex functions, $h_{1}: \mathbb{R}^{T} \rightarrow \mathbb{R}^{T_{1}}, \ldots, h_{n}=\mathbb{R}^{T} \rightarrow \mathbb{R}^{T_{n}}$ are linear functions. The main aim is to transform the problem into an equal matrix-free cone program and solve it by using a matrix-free cone solver.

Consider that the problem satisfies a set of requirement known as disciplined convex programming. The requirements guarantee that each $f_{0}$ and $f_{n}$ can be represented as partial minimization over a cone program. Consider each function $f_{h}$ have the cone program representation as:

$$
\begin{aligned}
f_{h}( & \left.\left\|A s_{o}-\left[\begin{array}{ll}
A T_{h} & I
\end{array}\right]\left[\begin{array}{c}
\tilde{e} \\
\tilde{u}
\end{array}\right]\right\|_{2}\right) \\
& =\min _{T_{h}} g_{0}^{h}\left(A s_{o}\right. \\
& \left.-\left[\begin{array}{ll}
A T_{h} & I
\end{array}\right]\left[\begin{array}{c}
\tilde{e} \\
\tilde{u}
\end{array}\right], T_{h}\right)+e
\end{aligned}
$$

Subject to $g_{j}^{h}\left(A s_{o}-\left[\begin{array}{ll}A T_{h} & I\end{array}\right]\left[\begin{array}{l}\tilde{e} \\ \tilde{u}\end{array}\right], T_{h}\right)+e \in$

$$
\mathcal{K}_{j}^{h}, j=1, \ldots, r^{h}
$$

In Eq. (16), $T_{h} \in \mathbb{R}^{T}$ is the optimization variable, $g_{0}^{h}, \ldots, g_{r^{h}}^{h}$ are linear functions and $\mathcal{K}_{1}^{h}, \ldots, \mathcal{K}_{r^{h}}^{h}$ are convex cones. The problem (15) can be rewritten as an equal cone program as follows:

$$
\min _{\tilde{e} \in \mathbb{R}^{T}, \widetilde{u} \in \mathbb{R}^{t+T-2}} f_{0}\left(\|\tilde{e}\|_{1}+\|\tilde{u}\|_{1}\right)
$$

Subject to $-g_{0}^{h}\left(A s_{o}-\left[\begin{array}{ll}A T_{h} & I\end{array}\right]\left[\begin{array}{l}\tilde{e} \\ \tilde{u}\end{array}\right], T_{h}\right)-e \in$

$$
\begin{aligned}
& \mathcal{K}_{+}, h=1, \ldots, n \text {, }
\end{aligned}
$$

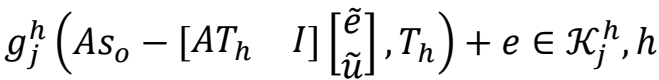

$$
\begin{aligned}
& =1, \ldots, n, j=1, \ldots, r^{h} \\
& h_{i} * \tilde{e}=T_{h} \tilde{e}, i=1, \ldots, n
\end{aligned}
$$

Moreover, the problem (17) is converted into a standard form for a matrix-free cone program in (14) 
by representing $f_{0}$ as the inner product with a vector $(l+c) \in \mathbb{R}^{T}$, concatenating $h_{i}$ and $e$ vectors into a single vector $b$ and representing the matrix $A$ implicitly as the linear function that stacks the outputs of all $h_{i}$ and $g_{j}^{h}$ excluding $g_{0}^{h}$ into a single vector. Thus, the optimal solutions of $\tilde{e}$ and $\tilde{u}$ are obtained by solving the convex optimization problem to estimate $e$ and $A s_{b}$. Finally, the true SCR events $(h * \tilde{e})$ are reconstructed.

\section{Experimental results}

In this section, performance efficiency of the SCR event detection from EDA signals using proposed MCSD model is evaluated by using MATLAB 2014a and compared with the existing sparse decomposition model [7], cvxEDA [9], CSD [3] model. In this experiment, WESAD dataset is considered that contains the data collected from 15 subjects with Trier Social Stress test as stress stimuli. The data has been recorded by using two different devices such as one chest-based and one wrist-based, each including high-resolution physiological and motion data. In this analysis, totally 427794 EDA signals are acquired in which 377794 signal samples are used for training and remaining 50000 samples are used for testing. The comparison is made in terms of different metrics such as precision, recall, accuracy and Receiver Operating Characteristic (ROC) curve.

The observed signal and the recovered signal and the result of signal decomposition on the $4 \mathrm{~Hz}$ down-sampled signal are shown in Fig. 1 and 2, respectively. In Fig. 2, the recovered signals using both CSD and MCSD models are highlighted without positivity constraints on the SCR events signal whereas in Fig. 3, the recovered signals using both CSD and MCSD models are highlighted with positivity constraints on the SCR events signal. The signal shown in Fig. 2 \& Fig. 3 is chosen randomly for any user from the WESAD dataset. Stimuli are presented to the user at moments denoted by brown dotted vertical lines. It is observed that the recovered SCR events signal is similar for both models. The reconstructed SCR signals $h * \tilde{e}$ using both models are also illustrated. In general, it is demonstrated that the proposed MCSD signal based SCR events detection performs similarly to its variation with positivity constraints.

\subsection{Precision}

It is computed based on the fraction of recovered and detected SCR signal events which are relevant at True Positive (TP) and False Positive (FP) rates.

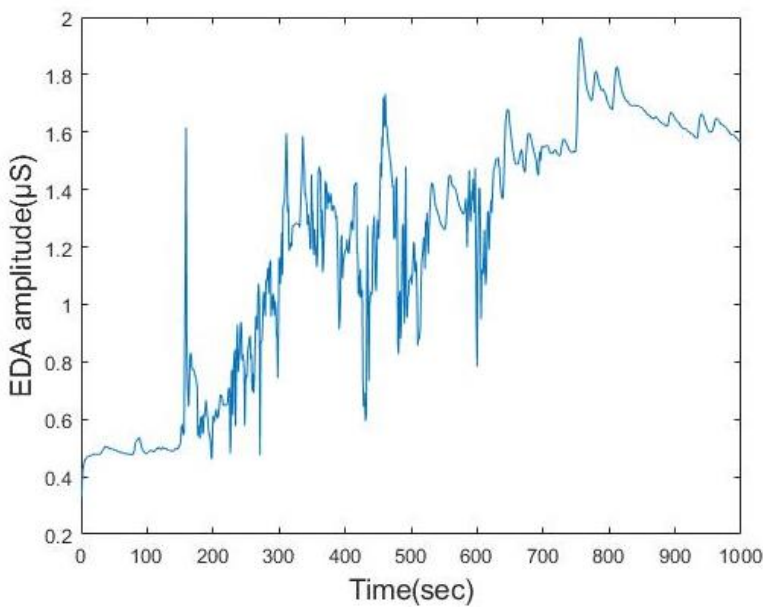

Figure.1 Original EDA signal

$$
\text { Precision }=\frac{T P}{T P+F P}
$$

Fig. 4 shows the comparison of both existing and proposed models using EDA signals for SCR events detection in terms of precision. From this analysis, it is observed that the proposed MCSD model achieves higher precision than the other model for EDA representation and decomposition. For example, the overall precision of MCSD for detecting true SCR events is $0.81 \%$ higher than all other models.

\subsection{Recall}

It is computed based on the fraction of recovered and detected SCR events at TP and False Negative (FN) rates.

$$
\text { Recall }=\frac{T P}{T P+F N}
$$

Fig. 5 shows the comparison of SCR events detection using both proposed and existing models in terms of recall. From this analysis, it is observed that the proposed MCSD model achieves higher recall than other existing models for detecting the SCR events from EDA signal. For instance, the overall recall of MCSD is $0.35 \%$ higher than all other models.

\subsection{Accuracy}

It is computed based on the TP and True Negative (TN) rates among the total number of SCR events detected.

$$
\text { Accuracy }=\frac{T P+T N}{T P+T N+F P+F N}
$$


Recovered SCR events

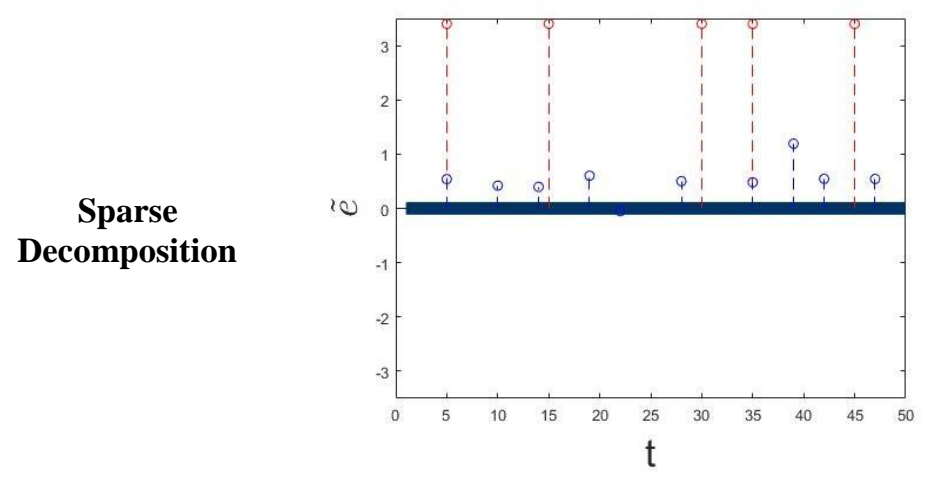

cvxEDA

CSD

MCSD
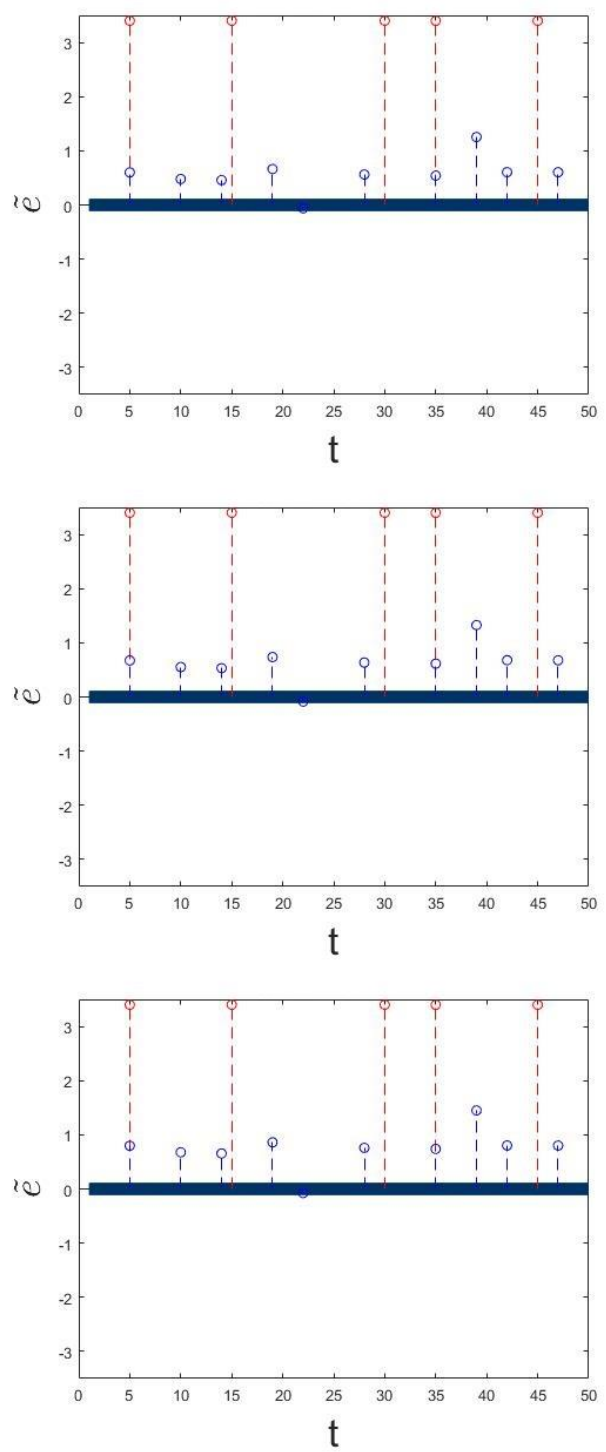

Recovered SCRs
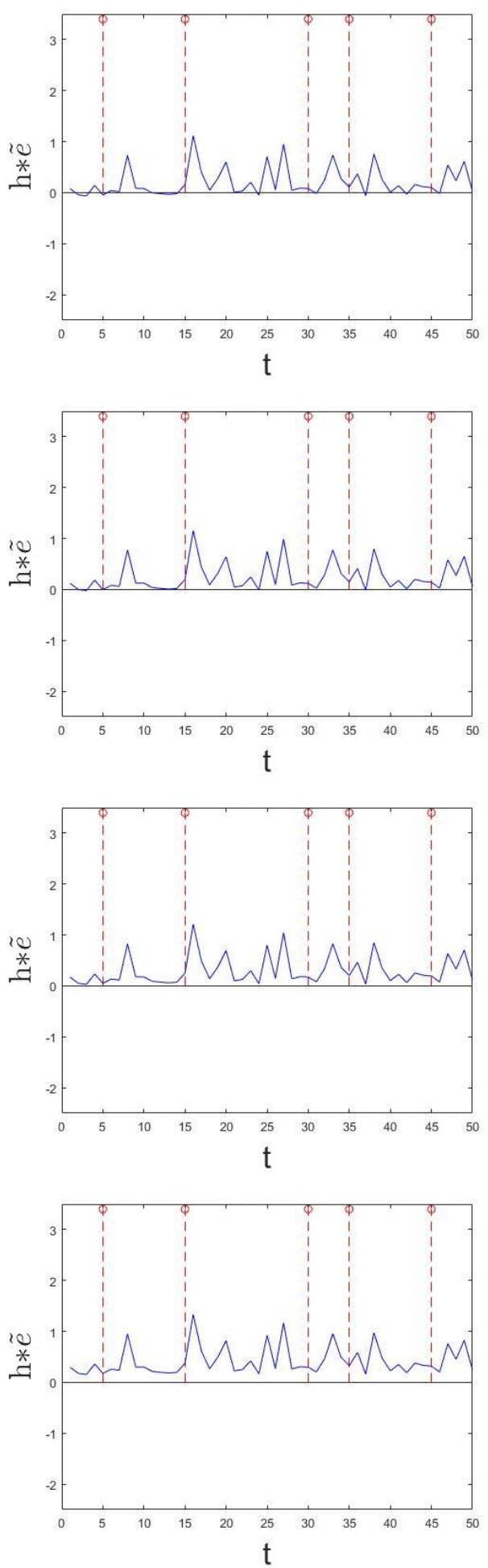

Figure.2 Results for proposed and existing models without Positivity Constraint for SCR event signal decomposition on the $4 \mathrm{~Hz}$ down-sampled signal 
Recovered SCR events with Positivity

Sparse Decomposition

cvxEDA

CSD

MCSD
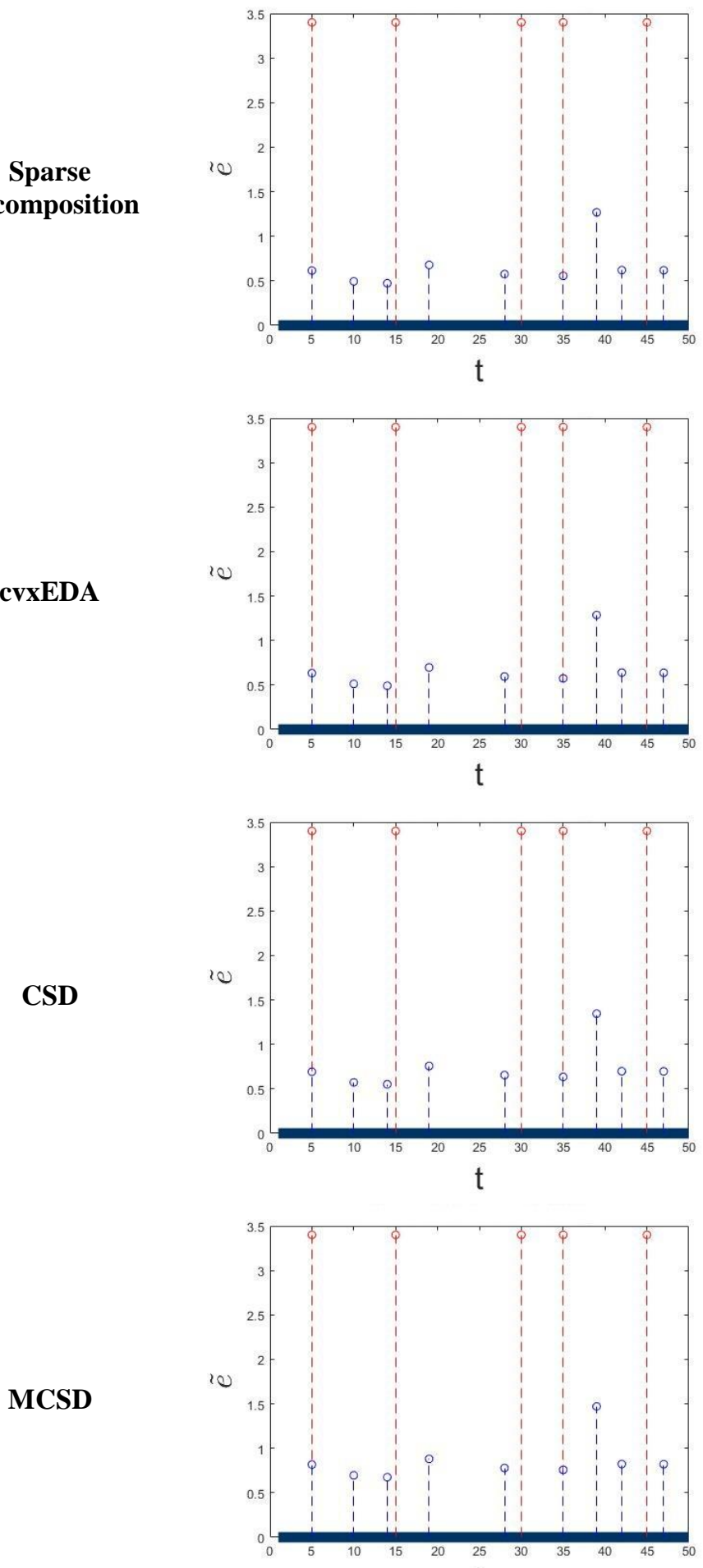

$\mathrm{t}$

\section{Recovered SCRs with Positivity}
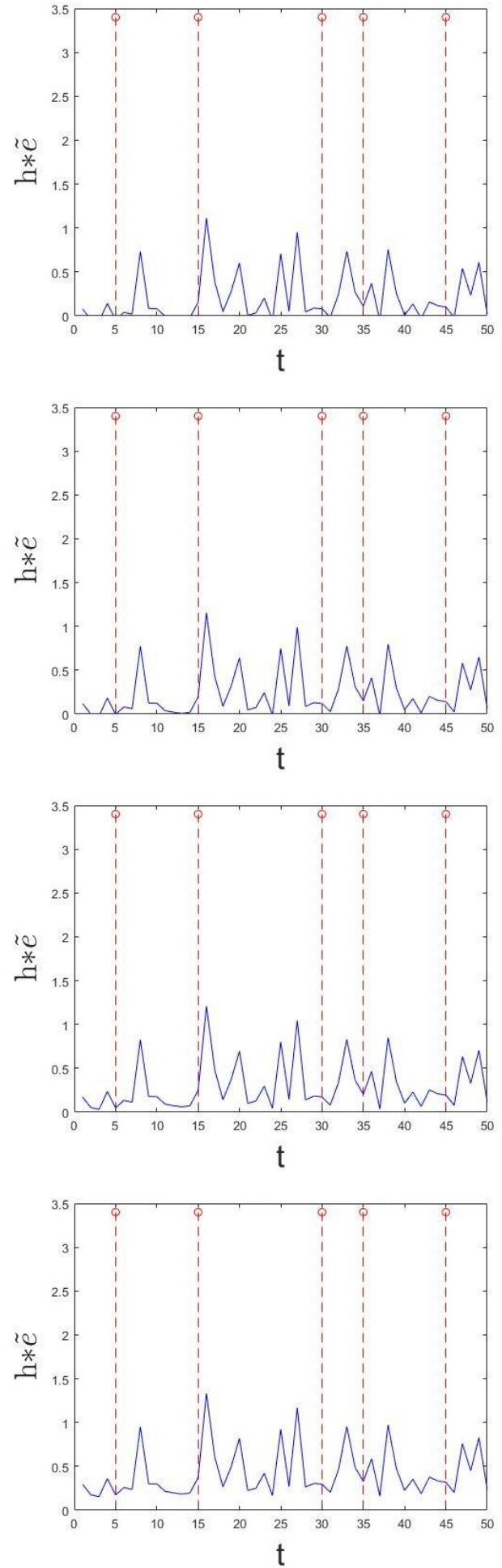

Figure.3 Results for proposed and existing models with Positivity Constraint for SCR event signal decomposition on the $4 \mathrm{~Hz}$ down-sampled signal 


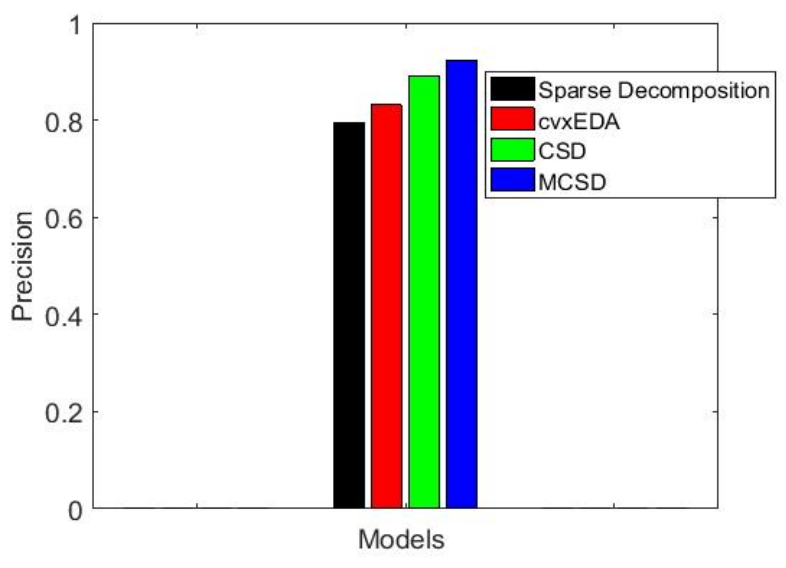

Figure.4 Comparison of precision

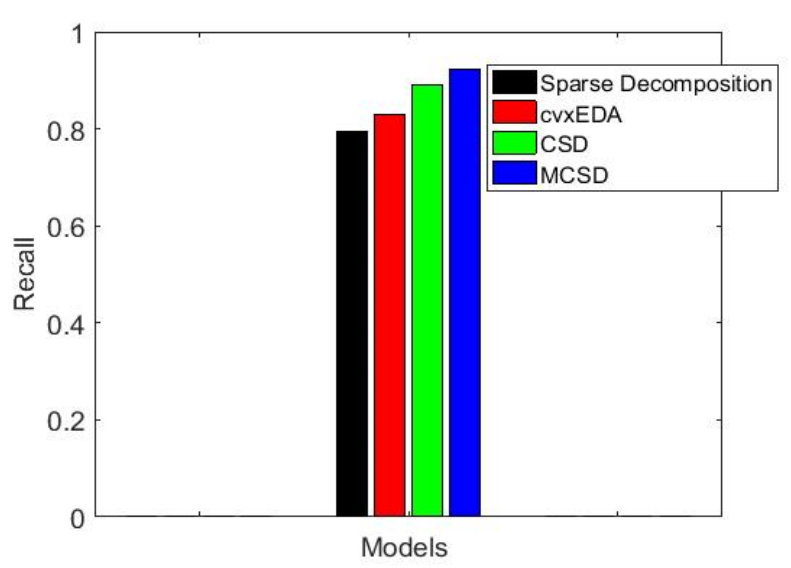

Figure.5 Comparison of recall

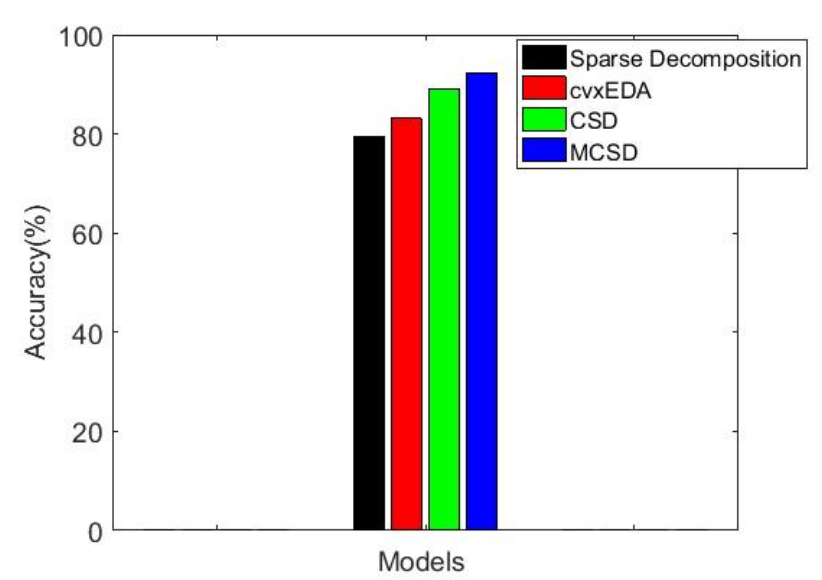

Figure.6 Comparison of accuracy

Fig. 6 shows the comparison of proposed and existing models used for SCR events detection in terms of accuracy (\%). From this analysis, it is observed that the proposed MCSD model achieves higher accuracy for detecting SCR events from the EDA signals compared to the other existing models. For example, the overall accuracy of MCSD is $2.55 \%$ higher than all other models.

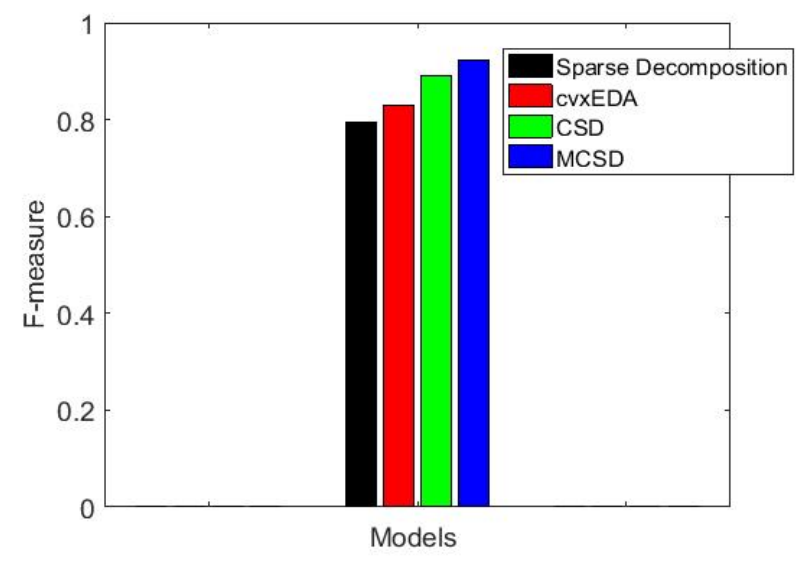

Figure.7 Comparison of f-measure

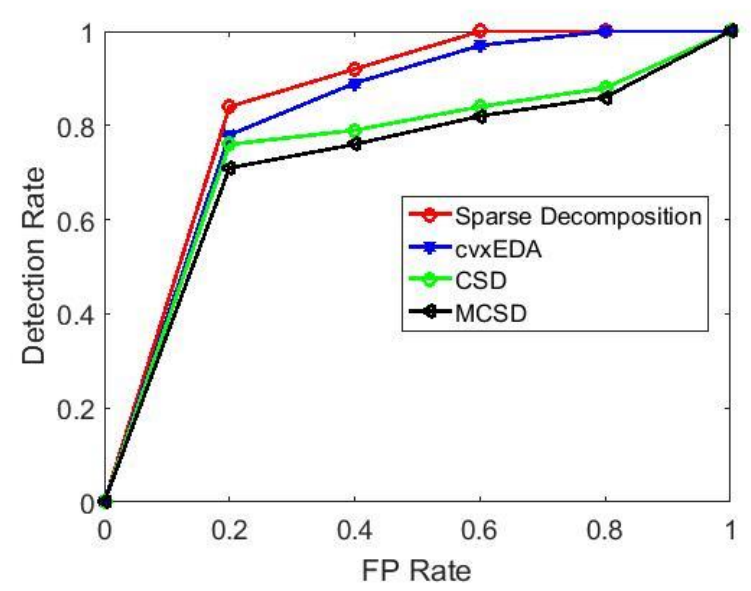

Figure.8 Comparison of ROC

\subsection{F-measure}

It is computed by using both precision and recall as:

$$
F-\text { measure }=2 \cdot\left(\frac{\text { Precision } \cdot \text { Recall }}{\text { Precision }+ \text { Recall }}\right)
$$

Fig. 7 shows the comparison of true SCR events detection using both proposed and existing models in terms of f-measure. From this analysis, it is observed that the proposed MCSD model achieves higher f-measure than the other existing models. For example, the overall $\mathrm{f}$-measure of MCSD model for SCR events detection using EDA signal is $0.82 \%$ higher than all other existing models.

\subsection{ROC analysis}

The ROC curve defines the relation between the FP rate and detection rate. Fig. 8 shows the comparison of ROC curves for SCR events detection using proposed and existing EDA decomposition models. From this analysis, it is 


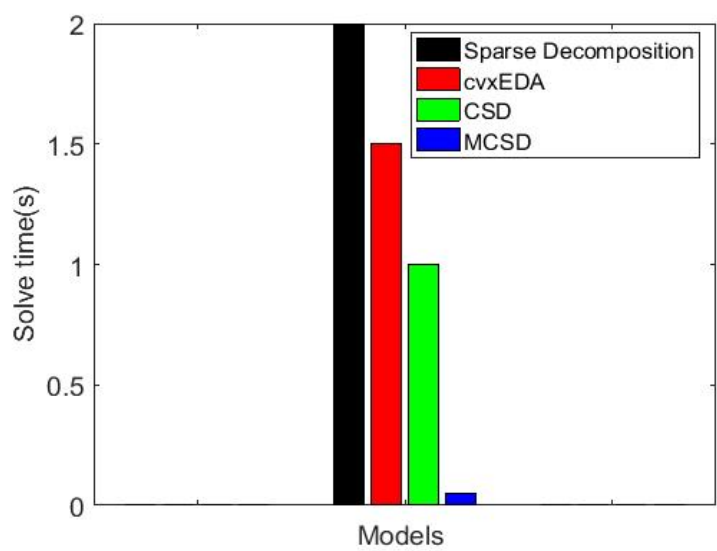

Figure.9 Comparison of computational complexity

observed that the proposed MCSD model achieves better recovery and detection of SCR events signal than other existing models. For example, consider the FP rate is 0.6. Then, the overall detection rate of MCSD model is 3.09\% higher than all other models.

\subsection{Computational complexity}

It is defined as the time taken to solve the optimization problem given in the EDA signal decomposition. This is analyzed based on the average solve time in seconds.

Fig. 9 shows the comparison of both proposed and existing models for SCR events detection using EDA signals in terms of computational complexity i.e., solve time taken in seconds. From this analysis, it is observed that the proposed MCSD model achieves less amount of time to solve the given optimization problem during EDA signal decomposition. Since this model uses matrix-free convex optimization to represent and decompose the EDA signal and detecting true SCR events. For example, the solve time of MCSD is $95 \%$ less than the other existing models.

Thus, it is proved that the proposed MCSD model for EDA signal achieves better precision, recall, f-measure and accuracy than the other existing models. Also, it achieves less computational complexity than the existing models. This is because of the utilization of matrix-free convex modeling which simplifies the optimization problem of obtaining the recovered SCR events and provides the recovered SCR events accurately.

\section{Conclusion}

In this article, a MCSD model for EDA signal is proposed for solving the problem while the baseline signal is present with the EDA signals. In this model, the shape of impulse response with time and different noise models may vary to improve the recovery accuracy and enhance the human stress monitoring system. This model has a matrix-free convex optimization model to solve the de-mixing problem that exploits the Toeplitz structure for facilitating the decomposition of EDA signals by means of provable bounds on the recovery of true SCR events. Finally, the experimental results proved that the detection of SCR events using proposed MCSD model achieves an accuracy of $92.4 \%$ which is $10.09 \%$ higher than all other existing models. Similarly, the precision, recall and f-measure values of MCSD model is nearly $0.82 \%$ higher than the other existing models. Thus, the proposed MCSD model can effectively decompose the EDA signal and detects the SCR events. The future extension of this work includes the automatic detection of artifacts and human activity classification from the EDA signal.

\section{References}

[1] D. Malathi, J. D. Jayaseeli, S. Madhuri, and K. Senthilkumar, "Electrodermal Activity Based Wearable Device for Drowsy Drivers", Journal of Physics: Conference Series, IOP Publishing, Vol. 1000, No. 1, p. 012048, 2018.

[2] R. Sioni and L. Chittaro, "Stress detection using physiological sensors", Computer, Vol. 48, No. 10, pp. 26-33, 2015.

[3] S. Jain, U. Oswal, K. S. Xu, B. Eriksson, and J. Haupt, "A compressed sensing based decomposition of electrodermal activity signals", IEEE Transactions on Biomedical Engineering, Vol. 64, No. 9, pp. 2142-2151, 2017.

[4] A. Finset, T. L. Stensrud, E. Holt, W. Verheul, and J. Bensing, "Electrodermal activity in response to empathic statements in clinical interviews with fibromyalgia patients", Patient Education and Counseling, Vol. 82, No. 3, pp. 355-360, 2011.

[5] C. Kappeler-Setz, F. Gravenhorst, J. Schumm, B. Arnrich, and G. Tröster, "Towards long term monitoring of electrodermal activity in daily life", Personal and Ubiquitous Computing, Vol. 17, No. 2, pp. 261-271, 2013.

[6] A. Sano, R. W. Picard, and R. Stickgold, "Quantitative analysis of wrist electrodermal activity during sleep", International Journal of Psychophysiology, Vol. 94, No. 3, pp. 382-389, 2014.

[7] T. Chaspari, A. Tsiartas, L. I. Stein, S. A. Cermak, and S. S. Narayanan, "Sparse representation of electrodermal activity with 
knowledge-driven dictionaries", IEEE Transactions on Biomedical Engineering, Vol. 62, No. 3, pp. 960-971, 2015.

[8] V. Xia, N. Jaques, S. Taylor, S. Fedor, and R. Picard, "Active learning for electrodermal activity classification", In: IEEE Signal Processing in Medicine and Biology Symposium, pp. 1-6, 2015.

[9] A. Greco, G. Valenza, A. Lanata, E. P. Scilingo, and L. Citi, "cvxEDA: A convex optimization approach to electrodermal activity processing", IEEE Transactions on Biomedical Engineering, Vol. 63, No. 4, pp. 797-804, 2016.

[10] P. Ghaderyan and A. Abbasi, "An efficient automatic workload estimation method based on electrodermal activity using pattern classifier combinations", International Journal of Psychophysiology, Vol. 110, pp. 91-101, 2016.

[11] L. Giromini, R. Morese, A. Salatino, M. Di Girolamo, D. J. Viglione, and A. Zennaro, "Rorschach Performance Assessment System (R-PAS) and vulnerability to stress: A preliminary study on electrodermal activity during stress", Psychiatry Research, Vol. 246, pp. 166-172, 2016.

[12] Melander, J. Martinsson, and S. Gustafsson, "Measuring Electrodermal Activity to Improve the Identification of Agitation in Individuals with Dementia", Dementia and Geriatric Cognitive Disorders Extra, Vol. 7, No. 3, pp. 430-439, 2017.

[13] S. H. Hwang, S. Seo, H. N. Yoon, D. W. Jung, H. J. Baek, J. Cho, J. W. Choi, Y. J. Lee, D.-U. Jeong, and K. S. Park, "Sleep period time estimation based on electrodermal activity", IEEE Journal of Biomedical and Health Informatics, Vol. 21, No. 1, pp. 115-122, 2017.

[14] R. Zangróniz, A. Martínez-Rodrigo, J. M. Pastor, M. T. López, and A. FernándezCaballero, "Electrodermal Activity Sensor for Classification of Calm/Distress Condition", Sensors, Vol. 17, No. 10, p. 2324, 2017. 